\title{
Assessing the WiFi offloading benefit on both service performance and EMF exposure in urban areas
}

\author{
Mathieu Brau ${ }^{1}$, Julien Stéphan ${ }^{1}$, Luis Diez ${ }^{2}$, Yoann Corre ${ }^{1}$, Yves Lostanlen ${ }^{3}$, Ramon Agüero ${ }^{2}$ \\ ${ }^{1}$ Siradel, St-Grégoire, France. \\ ${ }^{2}$ University of Cantabria, Santander, Spain. \\ ${ }^{3}$ Siradel North America, Toronto, Canada, \\ mbrau@siradel.com, \{ldiez,ramon\}@tlmat.unican.es
}

\begin{abstract}
In this paper we assess the benefit of WiFi offloading over dense urban scenarios in terms of both Quality of Service (QoS) and Electromagnetic Field (EMF) exposure. This study relies on results obtained with two complementary simulation platforms: a two-tier dynamic system-level simulator and a 3D coverage-based simulator. Outputs are usual service coverage key performance indicators, handover probability statistics, as well as common and innovative metrics for EMF exposure characterization that jointly take into account the contributions from the base-station and the User-Equipment (UE) transmissions. The main outcome is that, for elastic services, the best QoS and minimum global EMF exposure are jointly achieved with maximum WiFi offloading.
\end{abstract}

\section{Keywords-WiFi offloading, LTE, EMF exposure}

\section{INTRODUCTION}

In order to overcome the capacity limitation, operators are considering the use of non-cellular technologies like WiFi so as to offload mobile data traffic. The operator offloading policy, governed by Access Network Discovery and Selection Function (ANSDF), selects a set of services (e.g. video download) that shall be, as much as possible, transferred to $\mathrm{WiFi}$, to optimize the core network efficiency in terms of delay, capacity and cost. This technique is viewed as a very cost-effective solution to support important traffic increases in the cellular network with a limited investment in the infrastructure.

Along with the increment on mobile traffic demand, there has been an increasing concern from the users point of view about the electro-magnetic fields (EMF) exposure caused by mobile communications. In this regard, the LEXNET project [1] introduces an innovative approach to assess the exposure of a whole population, where both DownLink (DL) and UpLink (UL) contributions are summed up into the so-called Exposure Index (EI) [2]. In the scope of LEXNET, cellular traffic offloading has been identified as a promising technique to reduce the EMF exposure perceived by the end-users, without jeopardizing the perceived Quality of Service (QoS) [3].

In this work, both the service performance and global EMF exposure are evaluated in an urban offloading scenario. Two complementary simulation platforms are used. First, a dynamic system-level simulator offers a detailed modelling of network selection and resource allocation procedures based on services demand. By individually considering users and services, the tool is able to apply different access policies, carrying out a step-wise service analysis (see [4] for more details). Second, a coverage-based simulator predicts the user performance and the EMF metrics in a real environment and over large-scale 3D maps, based on abstracted models regarding the network selection. General statistics are extracted from those maps.

The remainder of this paper is structured as follows: Section II depicts the most relevant aspects of the coveragebased simulator, paying special attention to the $\mathrm{WiFi}$ prediction. Section III discusses how the offloading has been configured. Then, Section IV describes the main results: Section IV.A is devoted to the evaluation carried out with the coverage-based simulator, while the analysis (at the service level) performed with the system-level simulator is drawn in Section IV.B. Finally, Section V points out the most relevant conclusions extracted from this study.

\section{SIMULATOR PLATFORMS PRINCIPLE}

\section{A. Coverage-based simulator}

The coverage-based simulator generates outdoor and multifloor indoor throughput and EMF maps, from which network performance statistics are extracted.

The eNodeBs are placed on dominant locations in a real dense urban environment (part of Paris). The DL received powers from the eNodeBs to every pixel is calculated in a deterministic way, using a ray-based method. Each pixel of the simulation map is viewed as a possible user location. The LTE user peak throughputs, the per-cell resource allocations and the inter-cell interferences are simulated all together and using some simplifications, the reader may refer to [5] for further explanations.

A WiFi abstraction model is introduced that provides statistics on the WiFi DL/UL PHY peak throughputs available inside buildings in the $2.4 \mathrm{GHz}$ frequency band. The model provides statistics on both the WiFi air interface (PHY) throughput, and on the overall connection data rate, for which possible backhaul imitations (due to XDSL performance) are considered. Furthermore, the model determines the additional DL EMF exposure that is generated when the traffic in the WiFi Access Points (APs) layer is increased. The derivation of this abstraction model is further detailed below. 
The RAT selection module determines, for each user communication, whether it should be served by the LTE or WiFi network. The decision is based on: (1) the type of service, since only a subset of services is allowed to be offloaded; (2) the predicted LTE DL PHY throughput; and (3) a WiFi DL PHY throughput, given by a random generator that implements the WiFi abstraction model. In the proposed study, the communication is decided to be offloaded if the WiFi DL PHY throughput is higher than the LTE DL PHY throughput.

The tool also simulates the UL transmit power efficiency $(\mathrm{kbps} / \mathrm{W})$, defined as the ratio between the throughput and UL transmit power. This metric is inversely proportional to a dose figure (active level $\times$ duration) and it is used here as a joint measure of both the UL EMF exposure and the UL network performance. The LEXNET EI is also assessed, which merges the exposure incurred by personal devices with that attributable to base stations and APs. The EI is estimated by summing the Specific Absorption Rate (SAR) scaled by the exposure duration for each technology on both DL/UL radio links. It depends on inputs related to the population and network user properties that are not detailed here, but are available in [2].

\section{B. WiFi abstraction model}

The WiFi abstraction model has been derived from extensive simulations of a medium-size study area where the number of APs distributed in the different building floors is deduced from a typical $\mathrm{WiFi}$ penetration rate, i.e. 5000 private $\mathrm{AP} / \mathrm{km}^{2}$. The location of APs is drawn randomly, but with some constraints to prevent from having two APs in the same room (or "apartment"). The propagation loss is simulated from a Volcano ray-based model using parameters that were previously calibrated for the prediction of dense femto-cell deployments [6]. The propagation is computed inside the building where the AP is located, but also in surrounding streets and other buildings. The model was validated by comparison to in-street measurements collected in a European city center (Santander) from an existing but unknown WiFi deployment. The metric used in this comparison is the total beacon power, which is the sum of the beacon powers received from all detected APs. Fig. 1 shows the Cumulative Distribution Functions (CDF) built from respective measurements and simulations. Median values are very close and the RMSE (Root Mean Square Error) is only $6.8 \mathrm{~dB}$.

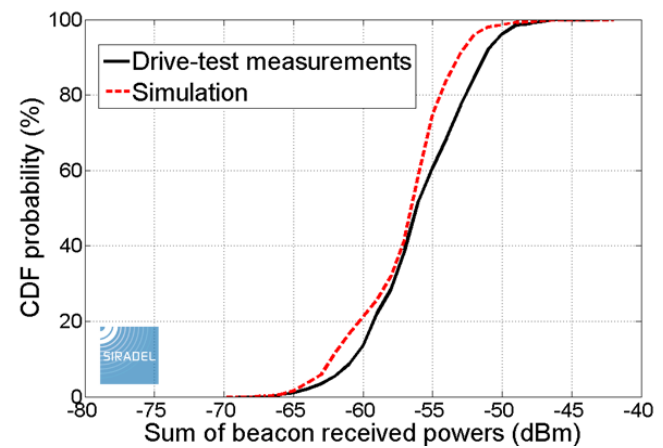

Figure 1: Comparison of total beacon power between simulation and measurements

\section{Dynamic system-level simulator}

The dynamic system-level simulator is complementary to the coverage-based simulator, in particular regarding the modeling of network selection and resource allocation procedures. The tool implements a set of analytical models for LTE coverage and resource allocation, and distance based model for WiFi technology [7]. More details about them can be found in [4]. This tool is fed with consecutive snapshots, which represent the status of the system, and therefore the access selection is applied in a discrete time operation; hence, the scenario dynamics and memory can be taken into account. This approach can be therefore exploited to compare different access policies and to rank them by comparison with the defined optimum behavior. We distinguish two types of service: the first one (Type 1) is allowed to connect to $\mathrm{WiFi}$ APs, while the second one (Type 2) is limited to the LTE cellular network. The users will try to connect to the access element, either eNodeB or AP that provides the best conditions: highest Reference Signal Received Power (RSRP) for LTE or highest raw data rate in the case of WiFi APs. Only if a WiFi connection is not possible, the user will try to use the LTE layer provided the access is granted.

\section{OFFLOADING SCENARIO}

The offloading study is conducted in a large dense urban environment, where both WiFi and LTE service coverages are quite broad. The LTE network is assumed to be highly loaded, but without reaching a saturation state, i.e. all users are served.

TABLE 1: OFFLOADING SCENARIO PARAMETERS

\begin{tabular}{|c|c|}
\hline LTE layout: & FDD 2×10 MHz @2.6 GHz \\
\hline $\begin{array}{l}\text { Macro cell } \\
\text { deployment }\end{array}$ & $\begin{array}{l}\text { Inter-site distance } 450 \mathrm{~m} \\
19 \text { tri-sector sites }\end{array}$ \\
\hline eNodeB & $\begin{array}{l}\text { Max transmit power: } 43 \mathrm{dBm} \\
\text { Directive antenna / } 14 \mathrm{dBi} / 6^{\circ} \text { downtilt } \\
\text { UL noise figure: } 2.5 \mathrm{~dB}\end{array}$ \\
\hline User Equipment (UE) & $\begin{array}{l}\text { UL transmit power: from }-40 \text { to }+23 \mathrm{dBm} \\
\text { Omni antenna / } 0 \mathrm{dBi} \\
\text { DL noise figure: } 9 \mathrm{~dB}\end{array}$ \\
\hline WiFi layout & 802.11n@2.4 GHz \\
\hline AP deployment & 800 indoor $\mathrm{AP} / \mathrm{km}^{2}$ \\
\hline AP & $\begin{array}{l}\text { Transmit power: } 20 \mathrm{dBm} \\
\text { Omni half-wave antenna / } 2.15 \mathrm{dBi} \\
\text { UL noise figure: } 6 \mathrm{~dB}\end{array}$ \\
\hline UE & $\begin{array}{l}\text { Transmit power: } 20 \mathrm{dBm} \\
\text { Omni antenna / } 0 \mathrm{dBi} \\
\text { DL noise figure: } 9 \mathrm{~dB}\end{array}$ \\
\hline Access & $\begin{array}{l}\text { Private AP } \\
\text { P\% APs allow for a shared access (only } 40 \% \text { of } \\
\text { fixed and air-interface bandwidth is shared) }\end{array}$ \\
\hline $\begin{array}{l}\text { Core network } \\
\text { connection }\end{array}$ & $\begin{array}{l}\text { Q\% APs connected to optical fiber } \\
\text { Others connected to xDSL }\end{array}$ \\
\hline xDSL mean DL thput. & $15 \mathrm{Mbps}$ \\
\hline \multicolumn{2}{|l|}{ Mobile user traffic } \\
\hline Data traffic & $35 \mathrm{Mbps} / \mathrm{km}^{2} \mathrm{DL}, 2 \mathrm{Mbps} / \mathrm{km}^{2} \mathrm{UL}$ \\
\hline Spatial distribution & $20 \%$ outdoors, $80 \%$ indoors (into several floors) \\
\hline \multicolumn{2}{|l|}{ Offloading rules } \\
\hline Policy & $\begin{array}{l}\text { Only video can be offloaded, i.e. max } 45 \% \text { of the } \\
\text { whole mobile traffic amount }\end{array}$ \\
\hline User location & $\boldsymbol{R} \%$ indoor users have a possible full AP access \\
\hline
\end{tabular}


The detailed simulation parameters are presented in Table 1. Two categories of mobile users are distinguished. Those belonging to the first group are assumed to be located at home with both fixed WiFi and mobile LTE access managed by the same operator. Consequently, they can be offloaded to the WiFi with full open access to the AP bandwidth; and the offloaded radio link is likely to benefit from high WiFi air interface quality. The users of the second category do not have full access to any close AP. Those users can only be offloaded to the WiFi thanks to the shared resources of an AP managed by the cellular operator. The WiFi air interface quality depends on the distance and propagation conditions between the user and the selected AP. The ratio between both user categories is set by the parameter $R$.

\section{EVALUATION RESULTS}

This section discusses the main results obtained with the two complementary tools. First, Section IV.A shows the analysis carried out by the $3 \mathrm{D}$ coverage-based tool, specially focused on EMF exposure metrics and overall generic QoS levels. Then, Section IV.B depicts the evaluation performed by the dynamic system-level tool, which focuses on the impact of the WiFi offloading over particular services. Both simulation setups rely on very similar assumptions, providing together accurate exposure estimation with both overall and per-service QoS figures. Finally, Section IV.C presents additional use cases based on a non-ideal $\mathrm{WiFi}$ layout.

\section{A. Coverage-based results}

The evaluation scenario assumes unrestricted WiFi broadband access, i.e. $Q=100 \%$ - all APs are connected to optical fiber - and $R=100 \%$ - all indoor mobile users have a full-access to a close WiFi AP. Fig. 2 gives the main results for the LTE-only network (used as the baseline scenario in this evaluation) and the LTE+WiFi networks, distinguishing the performance of each layer. The benefit of the WiFi offloading is strong on both the mean DL peak throughput (2.9 times higher) and the mean DL field strength (3.6 times lower) due to the joint reduction of the macro eNodeB transmission and the DL interference levels. The mean UL peak throughput for nonoffloaded users is slightly better compared to the baseline scenario because of a lower intra-LTE interference levels. The UL performance is much better for offloaded users (1.6 times higher), leading to a global improvement factor of 1.3.

Regarding the UL transmit power efficiency, the fixed WiFi transmit power $(20 \mathrm{dBm})$ makes the $\mathrm{WiFi}$ technology less efficient than LTE (with power control) on a comparable radio link. However, in our offloading scenario, users are much closer to the WiFi APs than LTE eNodeBs; therefore, the fixed WiFi transmit power does not imply a significant improvement in the overall UL transmit power efficiency: 1.6 times higher for indoor mobile users. Finally, as the WiFi offloading leads to both a strong DL field strength decrease and an improvement in the UL transmit power efficiency, the population EMF exposure measured by EI is significantly reduced: divided by 3.1 .
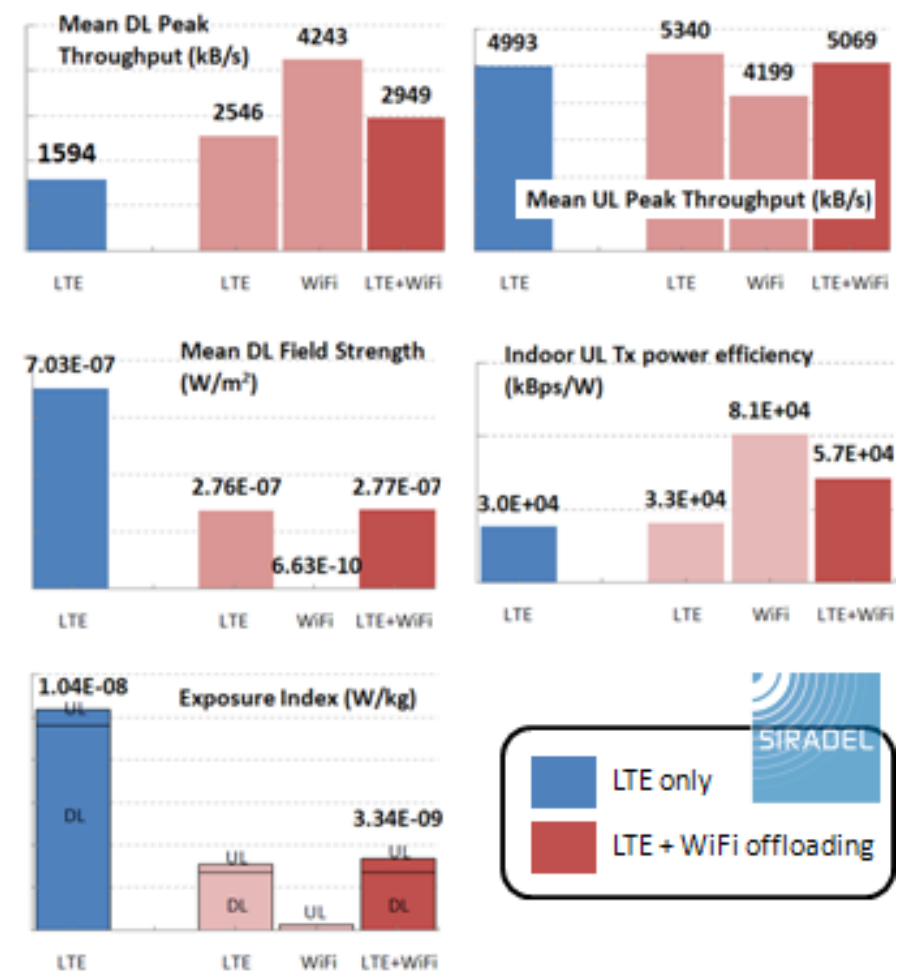

Figure 2: Network QoS and EMF performance before (blue) and after (red) the activation of WiFi offloading

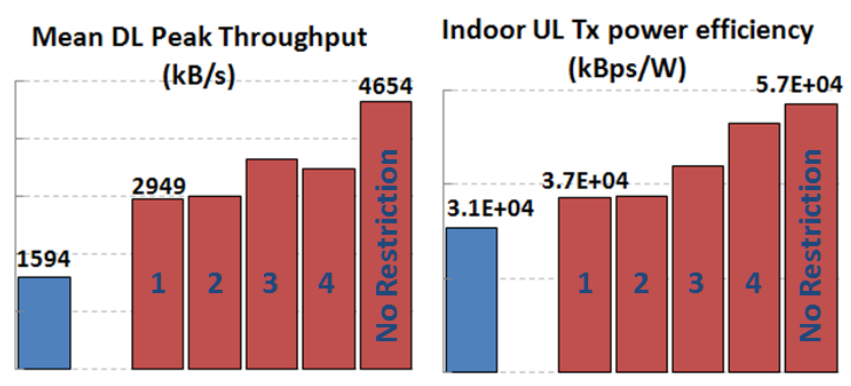

\begin{tabular}{|l|c|c|c|}
\hline Scenarios & $P$ & $Q$ & $R$ \\
\hline No restriction (section IV.A) & $100 \%$ & $100 \%$ & $100 \%$ \\
\hline 1) Non-ideal & $25 \%$ & $50 \%$ & $50 \%$ \\
\hline 2) $100 \%$ Shared access & $100 \%$ & $50 \%$ & $50 \%$ \\
\hline 3) $100 \%$ Optical fiber & $25 \%$ & $100 \%$ & $50 \%$ \\
\hline 4) $90 \%$ Full access users & $25 \%$ & $50 \%$ & $90 \%$ \\
\hline
\end{tabular}

Figure 3: Performance in different offloading scenarios

Other scenarios with non-ideal WiFi properties, thought to be closer to reality, have been simulated. The results in Fig. 3 show how the WiFi deployment and properties impact the offloading performance. Additional results are available in [8].

\section{B. System level results}

Despite the fact that approaches followed by both tools are different, the main characteristics of the scenario hold. Other scenario characteristics are depicted in Table 2. Users move according to a Random Waypoint model with a speed randomly selected within $[1,3] \mathrm{m} / \mathrm{s}$ (pedestrian). Each user is able to initiate two types of services, heavy and light, following an ON-OFF model with different parameters. As explained in Section II, Type 1 services are allowed to connect to WiFi APs, 
while those belonging to Type 2 are limited to the LTE cellular network. The first parameter we study is the percentage of unsuccessful services, which might be due to either the saturation of the access elements or to lack of access (coverage) to any element (i.e. outage). Fig. 4 shows the average failure probability for different number of deployed APs and for the different types of services: video and internet with and without the possibility to connect to WiFi APs, according to the scenario description. As can be observed, regardless of the offloading features, heavier services (i.e. video) are more likely to fail, since they have a longer duration and they require higher demand.

TABLE 2: SYSTEM LEVEL SCENARIO

\begin{tabular}{|l|l|l|l|l|}
\hline \multirow{3}{*}{ Services } & Type & Traffic & On & Off \\
\cline { 2 - 5 } & Video $(\mathrm{V})$ & $1000 \mathrm{Kbps}$ & $300 \mathrm{~s}$ & $1200 \mathrm{~s}$ \\
\cline { 2 - 5 } & Internet (I) & $64 \mathrm{Kbps}$ & $60 \mathrm{~s}$ & $80 \mathrm{~s}$ \\
\hline \multirow{3}{*}{ Traffic } & Type & Services & Usage & Access \\
\cline { 2 - 5 } & 1 & $\mathrm{~V}+\mathrm{I}$ & $45 \%$ & All networks \\
\cline { 2 - 5 } & 2 & $\mathrm{~V}+\mathrm{I}$ & $55 \%$ & Only cellular \\
\hline
\end{tabular}

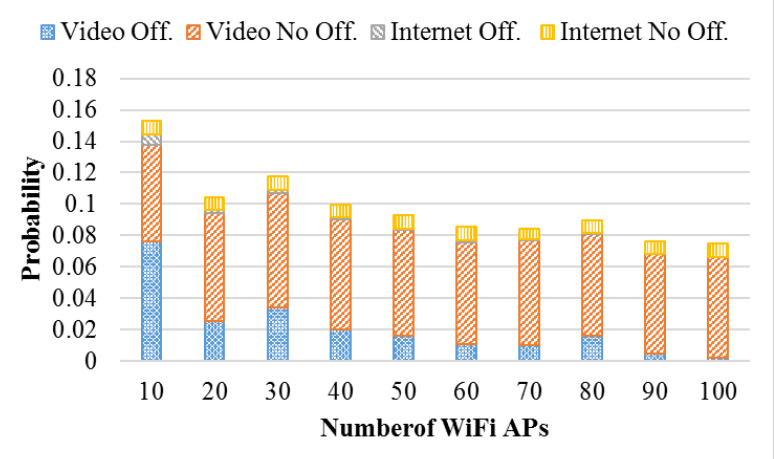

Figure 4: Probability of service failure vs. the number of APs

$\square$ Video WiFi Video LTE $₫$ Internet WiFi $\square$ Internet LTE

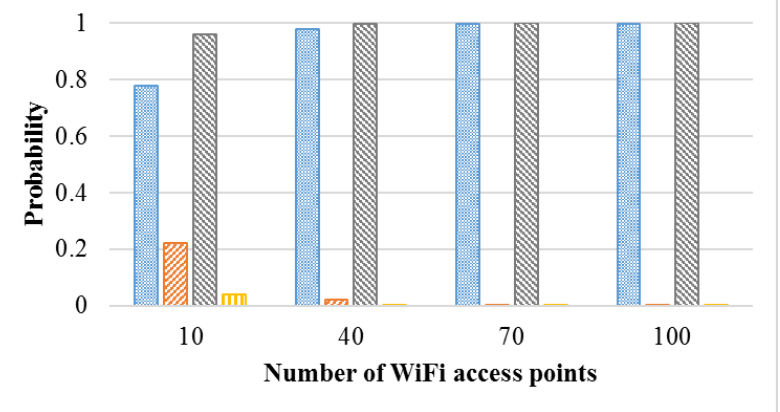

Figure 5: Ratio of time connected to each technology for offloading services

Furthermore, it is also shown that those services when they do not have the possibility of performing offloading, suffer from a higher failure probability, leading to a poorer QoS. Last, if we increase the number of deployed APs, the failure probability of those services that are allowed to use WiFi connections drastically decreases. Interestingly, the failure probability of those that cannot be offloaded to the WiFi layer is not heavily impacted by the presence of WiFi. This behavior can be explained by the fact that the service failure is mostly due to the user outage, since LTE eNodeBs do not get saturated in the considered scenario.
Next, Fig. 5 shows the time a particular service is connected to each technology. This result is only shown for those services that can be offloaded. This parameter is defined as the ratio of the time a service is connected to a particular technology and the total time the service is active; hence, the service failure probability has indeed an impact on this metric. Overall, it is observed that, with a low number of APs, services with longer duration relatively spend more time connected to the LTE network. On the other hand, services with shorter duration are able to stay connected to WiFi even with a lower number of APs.

\section{CONCLUSION}

By means of two complementary simulation platforms, a fine assessment of the WiFi offloading benefit in a dense urban scenario has been discussed in this paper. As expected, offloading allows for a significant improvement of DL and UL peak user throughputs: 2.9 and 1.3 times higher, respectively, in one of our scenarios. Furthermore, services that can be offloaded to the WiFi layer show a stronger robustness against failure, even when more horizontal handovers are required.

Besides, the paper demonstrates how WiFi offloading can highly improve the capacity together with a strong reduction of the population EMF exposure (reduced by a factor of 3.1 in the best scenario).

\section{ACKNOWLEDGMENT}

This paper reports work undertaken in the context of the FP7 project LEXNET (GA no 318273). Ramón Agüero also acknowledges the Spanish Government for the project "Connectivity as a Service: Access for the Internet of the Future", COSAIF (TEC2012-38574-C02-02).

\section{REFERENCES}

[1] M. Tesanovic and al., "The LEXNET Project: Wireless Networks and EMF: Paving the Way for Low-EMF Networks of the Future", IEEE Vehicular Technology Magazine, vol. 9, no. 2, pp. 20-28, June 2014

[2] N. Varsier and al., "A novel method to assess human population exposure induced by a wireless cellular network", Biolectromagnetics, vol. 36, Issue 6, pp 451-463, Sept. 2015.

[3] M. Popovic, M. Tesanovic, B. Radier, "Strategies for reducing the global EMF exposure: Cellular operators perspective", 11th International Symposium on Wireless Communications Systems (ISWCS), 2014.

[4] L. Diez, Y. Corre, B. Radier, M. Brau and R. Agüero. "Complementing radio-planning tools to analyze EMF-aware access selection", in Twelfth International Symposium on Wireless Communication Systems (ISWCS), 2015

[5] F. Letourneux, Y. Corre, E. Suteau, and Y. Lostanlen, "3D performance analysis of a heterogeneous LTE network with indoor small-cells in a real urban environment", IEEE International Conference on Communications (ICC), 2013.

[6] Y. Corre, J. Stéphan and Y. Lostanlen, "Indoor-to-outdoor path-loss models for Femtocell predictions”, 22nd IEEE PIMRC, Toronto, 2011.

[7] B. Coll, J. Gonzálvez, J. Sánchez Soriano, "Empirical performance models for P2P and two-hops multi-hop cellular networks with mobile realys", in Proceedings of the $8^{\text {th }}$ ACM workshop on performance monitoring and measurements of heterogeneous wireless and wired networks (PM2HW2N), 2013.

[8] Y. Corre and al. LEXNET D6.2: Report on Validation, 2015, available at: http://www.lexnet-project.eu/. 